\title{
Effects of discriminability, probability of reinforcement, and handling cost on visual search and prey choice
}

\author{
DONNA M. LACOURSE and DONALD S. BLOUGH \\ Brown University, Providence, Rhode Island
}

\begin{abstract}
Pigeons searched for two targets that varied in similarity to items in the background. The targets, simulating "prey," were small alphabetic characters presented on computer monitors among distractor characters. In the first experiment, the probability of reinforcement for pecking at the targets was manipulated. In the second experiment, the response requirement for one of the targets was varied. Changing the probability of reinforcement led to more efficient search and increased choice for the more reinforced item. This effect carried over to subsequent equal reinforcement baseline conditions. Increasing the response requirement for one item increased response time for that item and reduced choice of the item. This effect did not carry over to a subsequent baseline condition. The results suggested that a high probability of reinforcement resulted in improved detection of the item, perhaps through perceptual learning, and increased the incentive that motivated response to the item, but that high response requirement resulted only in an incentive shift.
\end{abstract}

Foraging in animals has often been viewed as governed by a cost-benefit tradeoff. Benefit is typically measured in terms of the amount of caloric energy gained from the prey item. Cost is measured in terms of amount of time and energy expended for search, recognition, capture, and consumption of a prey item. Optimal foraging models propose that an animal will choose a diet to maximize benefit and minimize cost, thus maximizing its overall fitness or the overall amount of energy gained (Schoener, 1987). Four variables that affect this cost-benefit computation are encounter rate, crypsis, prey quality, and handling cost. Search cost is directly affected by two of these variables, encounter rate and crypsis. As prey density increases, so will the encounter rate, and search cost will decline (Krebs, 1973). As prey crypsis increases (its discriminability declines), it may be misidentified, overlooked, or require a longer recognition time, all leading to increased search cost (Hughes, 1979).

Profitability is related to the quality of the prey item and its handling cost. Prey quality is equal to the amount of energy gained by consuming the item. Handling cost is a function of the amount of time and energy necessary for capturing, subduing, and consuming a prey item. Profitability is equal to prey quality divided by handling cost, so highly profitable prey have a high quality, a low handling cost, or both (Krebs, 1973). In simulated forag-

This research was supported by National Science Foundation Grant BNS-9407269. We would like to thank Patricia M. Blough for her comments on the manuscript. Address correspondence to D. S. Blough, Department of Psychology, Box 1853, Brown University, Providence, RI 02912 (e-mail: dblough@brown.edu).

-Accepted by previous editor, Robert A. Rescorla ing situations, "capture" of a prey item may yield food reinforcement only intermittently, and it is assumed that the quality of a prey item is directly related to its probability of reinforcement. Thus, if one item has a higher probability of reinforcement than another has, and if both have equal handling times, the first prey item will be more profitable than the second.

A number of operant studies (e.g., Abarca \& Fantino, 1982; Hanson \& Green, 1989; Lea, 1979) have examined how changes in quality and cost affect prey choice. In the cited studies, different response keys were used to simulate stages of foraging (search, choice, and handling) and different color keylights simulated different prey items. The experimenters manipulated quality and cost by varying the reinforcement and response contingencies. These studies generally supported qualitative predictions of optimal foraging theory, showing, for example, that as search cost increased, lower quality items were chosen more often; that is, specialization for the higher quality item decreased. However, as Shettleworth (1989) forcefully pointed out, questions about foraging optimality differ fundamentally from questions about the psychological processes that underlie choice. Studies such as those just cited were not designed to explore such processes. They could not show, for example, whether changes in profitability alter an animal's attention to or perception of prey, affect memory for prey, operate through the incentive value attached to different prey, or entail all of these and perhaps other mechanisms.

A number of experimenters have explored the psychological processes involved in tasks that simulate visual search for cryptic prey. Pietrewicz and Kamil (1981) used slides showing one of two types of moths on backgrounds in which the moths looked either cryptic or conspicuous. 
Similarly, Bond and Riley (1991) manipulated crypsis with slides that showed different sorts of grain on various backgrounds. Dawkins (1971a), Lawrence (1985a, 1985b, 1986), and Gendron (1986) used actual food items that were dyed either to match the background (cryptic) or to be conspicuous on the background. A common finding was that repeated encounters with a cryptic item led to more efficient detection of that item. However, profitability was not manipulated in these studies, and because only one type of prey, either cryptic or conspicuous, was available at one time, choice between types could not be assessed.

P. M. Blough (1992) manipulated crypsis by using three target characters (on a display monitor) that differed in their similarity to alphanumeric distractor items. On most trials, only one target appeared, whereas on some (choice trials), two of the three target items could appear simultaneously. She found that search time decreased when the targets were relatively conspicuous and when they were relatively frequent. On choice trials, the birds were more likely to choose the frequent item only if it was also the more discriminable of the two presented. This study showed that experience with different prey types influences search strategies and that choice is affected by both crypsis and encounter rate, but it did not show how profitability may interact with these variables.

As this brief summary suggests, effects of profitability on prey choice are well documented, but laboratory studies of prey profitability have not generally attempted to explicate the psychological processes underlying these effects. On the other hand, studies concerned with the processes underlying crypsis and frequency effects in search have not manipulated profitability. In the present experiments, we used the search method of P. M. Blough (1992) to explore effects of profitability variations on search for cryptic prey in a manner that might shed some light on underlying processes. We varied profitability in two ways. In the first experiment, we manipulated prey quality by changing the probability of reinforcement associated with each target item. In the second experiment, we manipulated handling cost by changing the number of pecks to the target that were required in order to end a trial. In both experiments, we also varied crypsis by using target items that differed in their similarity to background distractor items. Throughout, search speed and accuracy were recorded, and probe trials in which both target items were simultaneously presented were used to examine prey choice.

\section{EXPERIMENT 1}

In this experiment, we varied the relative quality of target items that differed in discriminability. Two targets appeared among an array of distractors. Quality was manipulated by varying the probability that responding to an item would produce food; discriminability was manipulated by making one target more similar to the distractors than the other. Handling cost remained constant and equal for both items.

\section{Method}

\section{Subjects}

Five male White Carneaux pigeons served as subjects. Three of the birds had previously served as subjects in a similar visual search experiment. The other 2 birds had participated in various autoshaping and delayed nonmatching-to-sample tasks.

The birds were maintained at approximately $80 \%$ of their freefeeding weights; a bird was not run if its weight exceeded 20 g more than that value. The pigeons had free access to water and typically received all their daily food during the experimental session.

\section{Apparatus and Stimulus Displays}

Three subject chambers, $34 \times 30 \times 34 \mathrm{~cm}$, were housed within three experimental boxes. White noise was delivered through a loudspeaker near the chambers. The front panel of each chamber contained two openings. One opening provided access to a monochromatic video monitor; the visible part of the monitor screen measured $12 \times 8.5 \mathrm{~cm}$. The top and bottom of the space were lined with pairs of infrared photoemitters and detectors that permitted the localization of pecks to the screen within six vertical strips. The second opening was centered $5 \mathrm{~cm}$ below the first and provided access to a magazine that delivered mixed grain. Three IBM XT computers controlled experiments and recorded response data.

The stimulus displays were composed of black letters or symbols on a white background. Though their dimensions varied with their shape, each item was approximately $4 \mathrm{~mm}$ high. The display items were arranged in four irregular rows consisting of six vertically aligned columns for a total of 24 possible stimulus locations. Each column corresponded to one of the six screen segments within which pecks were localized.

Test displays contained one of two possible targets amidst 23 distractor items. The target item could appear in any one of the 24 possible locations. The distractor items were identical to each other. The two targets differed in their similarity to the distractor; for convenience, the more cryptic (more similar) target is always "Target 1 " in the data analysis. During Phase 1 , the cryptic target item was a delta $(\delta)$ and the conspicuous target was the letter T. The distractor items were ampersands (\&). In Phase 2, the targets were the letters $\mathrm{O}$ (cryptic) and $\mathrm{E}$ (conspicuous), and the distractors were Qs. The first condition of each phase was used to confirm the difference in discriminability of the two targets.

\section{Procedure}

The same procedure was used in both the training and testing of Phases 1 and 2 except for the difference in target-distractor display items and the order of the reinforcement manipulations.

Training and response contingencies. All birds were first autoshaped to peck the target items from Phase 1. Once the birds had begun pecking reliably at the video monitor, a peck response requirement was initiated. To terminate a trial, the pigeon had to deliver an uninterrupted sequence of three pecks to the target location. Reaction time (RT) was recorded as the first peck in this sequence. If the sequence was interrupted-for example, if the bird pecked at one segment twice and then pecked at a new segment - then the initial RT was discarded, and RT for the first peck in the next uninterrupted series was recorded. Therefore, a "response" on any given trial was defined by a sequence of three uninterrupted pecks, and RT was recorded as the timc of the first peck in that sequence. Trials were separated by a 2 -sec intertrial interval (ITI) during which the screen was a blank white, the same color as the background of the stimulus display.

The probability of either target's appearing in the display was .5 , and each target had a probability of reinforcement of 1 . If the bird responded to the screen segment that contained the target item, a correct response was recorded, and the screen darkened temporarily. If no reinforcement was scheduled, the screen then turned white, and the ITI began. If reinforcement was to be delivered, the maga- 
zine opened as the screen turned white. Magazine time was adjusted for each bird so as to maintain it at $80 \%$ free-feeding weight.

If the bird responded to an incorrect segment of the screen, the display disappeared, the ITI was initiated, and a correction trial followed. The correction trial contained the same stimulus display as that on the preceding incorrect trial. This procedure continued until the bird made a correct response. Data from these correction trials were not included in the data analysis, nor did these trials count toward the session total.

During initial training for each phase, there were 60 trials per session, and no distractor items were used. When the pigeons were responding with $80 \%$ or greater accuracy, the probability of reinforcement for both target items was decreased by half, and the number of trials per session was doubled. When the probability of reinforcement was equal to .25 and accuracy was greater then $80 \%$, one distractor item ( $\&$ in Phase 1 or $\mathrm{Q}$ in Phase 2 ) was added to the display. The probability was again decreased, and the number of distractor items was doubled each time the bird responded with greater than $80 \%$ accuracy during a session. By the end of training, the total number of trials per session was 600 , the probability of reinforcement for each target was .1, and the total number of distractors was 23 .

Phase 1 and 2 testing. Phases 1 and 2 differed both in the targetdistractor combinations used and in the order of the probability of reinforcement manipulations used. Phase 1 was composed of five conditions in which the probability of reinforcement associated with each target was varied. Phase 2 was composed of three similar conditions and was conducted to provide further information on a carryover effect found in Condition 3 of Phase 1 (see below). Table 1 shows the probabilities associated with each target in the different conditions of Phases 1 and 2. During odd-numbered conditions (1, 3 , and 5), the probabilities of reinforcement associated with each target were equal. During even-numbered conditions ( 2 and 4 ), the two targets were associated with unequal probabilities of reinforcement.

The testing procedure was similar to training, except that probe trials were added and the target stimuli were differentially reinforced. On probe trials, both targets appeared in the stimulus display, although never in the same screen segment. The probability of a probe trial (shown in Table 1) was varied across conditions so that each session contained approximately 25 probe trials. These trials were never followed by correction trials, and the probability of reinforcement for a correct response on a probe trial was . 1 in all conditions of Phases 1 and 2 . The response requirement was a sequence of three uninterrupted pecks and the ITI was $2 \mathrm{sec}$ in all conditions of Phases 1 and 2.

Each condition was run for at least 11 sessions, continuing until the bird's performance had stabilized. Stability was defined as at least 4 consecutive sessions in which median RTs for both targets were neither continually increasing nor decreasing. Once stable per-

Table 1

Parameters for Phases 1 and 2 of Experiment 1

\begin{tabular}{ccccc}
\hline Condition & $\begin{array}{c}\text { Trials per } \\
\text { Session }\end{array}$ & $\begin{array}{c}\text { Probability } S^{\mathbf{R}} \\
\text { Targ1 }\end{array}$ & $\begin{array}{c}\text { Probability } S^{\mathrm{R}} \\
\text { Targ2 }\end{array}$ & $\begin{array}{c}\text { Probability } \\
\text { Probe }\end{array}$ \\
\hline \multicolumn{5}{c}{ Phase 1 } \\
1 & 625 & .1 & .1 & .04 \\
2 & 475 & .2 & .05 & .056 \\
3 & 325 & .2 & .2 & .083 \\
4 & 475 & .05 & .2 & .056 \\
5 & 625 & .1 & .1 & .04 \\
& & Phase 2 & & \\
1 & 625 & .1 & .1 & .04 \\
2 & 475 & .2 & .05 & .056 \\
3 & 625 & .1 & .1 & .04 \\
\hline
\end{tabular}

Note $-S^{R}$, probability of reinforcement for that target. The targets used in each phase were as follows: Phase 1, Targ1 was $\delta$ and Targ2 was T; Phase 2, Targ 1 was $\mathrm{O}$ and Targ2 was E. formance was established, the next 6 sessions were used for data analysis. Unless otherwise mentioned, a session was not included if overall accuracy was below $80 \%$.

\section{Results and Discussion}

The median RTs for the last six sessions of each condition were averaged for each bird in Phase 1. Figure 1A shows the mean of these values across birds. RT for the more cryptic target was greater than for the conspicuous target when the probability of reinforcement for both targets was equal (Condition 1). This difference reversed when the probability of reinforcement increased for the cryptic target and decreased for the conspicuous target, and this reversal carried over to Condition 3 , where both targets again had equal probabilities of reinforcement. When the probability of reinforcement was decreased for the cryptic target and increased for the conspicuous target (Condition 4), the difference in RTs reversed again, and this reversal carried over to the next condition, Condition 5 , when the probabilities were equal again.

Figures $1 \mathrm{~B}$ and $1 \mathrm{C}$ show that the accuracy and probe choice data mirrored the RT data: When RT was lower for one target than for the other, accuracy was higher for that target, and that target was chosen on a greater proportion of probe trials.

The statistical significance of these apparent effects was tested using within-subjects analyses of variance (ANOVAs) and Tukey HSD tests. The first analysis tested the effect of condition (i.e., profitability shifts) on the differences between cryptic target (Targ1) and conspicuous target (Targ2) scores. Results were considered significant at the $p \leq .05$ criterion level for both types of statistical analyses. The effect of condition was significant for the RT data $[F(4,16)=10.88]$, the accuracy data $[F(4,16)=14.79]$, and the proportion of conspicuous target (Targ2) choices on probe trials $[F(4,16)=11.96]$. By the Tukey HSD tests, Conditions 2 and 3 were significantly different from all other conditions except each other in terms of RT data $\left(\alpha=.05, M S_{\mathrm{e}}=39,488.05\right)$, accuracy data $\left(\alpha=.05, M S_{\mathrm{e}}=6.36\right)$, and the proportion data $\left(\alpha=.05, M S_{\mathrm{e}}=.0136\right)$.

A second analysis tested the effect of condition on the scores for each individual target type. The effect of condition on RT was significant for both target types [Targ1, $F(4,16)=3.44$, and $\operatorname{Targ} 2 F(4,16)=8.71]$, and the effect on accuracy was significant for Targ $1[F(4,16)=$ 3.82]. The following differences were significant by Tukey HSD tests: RTs from Conditions 1 and 3 differed for Targ $1\left(\alpha=.05, M S_{\mathrm{e}}=55,234.34\right)$, RTs from Conditions 4 and 5 differed from both Conditions 2 and 3 for Targ2 $\left(\alpha=.05, M S_{\mathrm{e}}=23,615.81\right)$, and Condition 2 differed in accuracy from Condition 5 for Targ $1(\alpha=.05$, $M S_{\mathrm{e}}=9.26$ ).

Figure 2 summarizes mean RT, mean accuracy, and mean proportion of Targ2 choices on probe trials for all 5 birds in Phase 2. As in Phase 1, the RT was greater for the cryptic target than for the conspicuous target when the probabilities of reinforcement were equal, as can be seen in Figure 2A. This difference in RTs reversed in Con- 

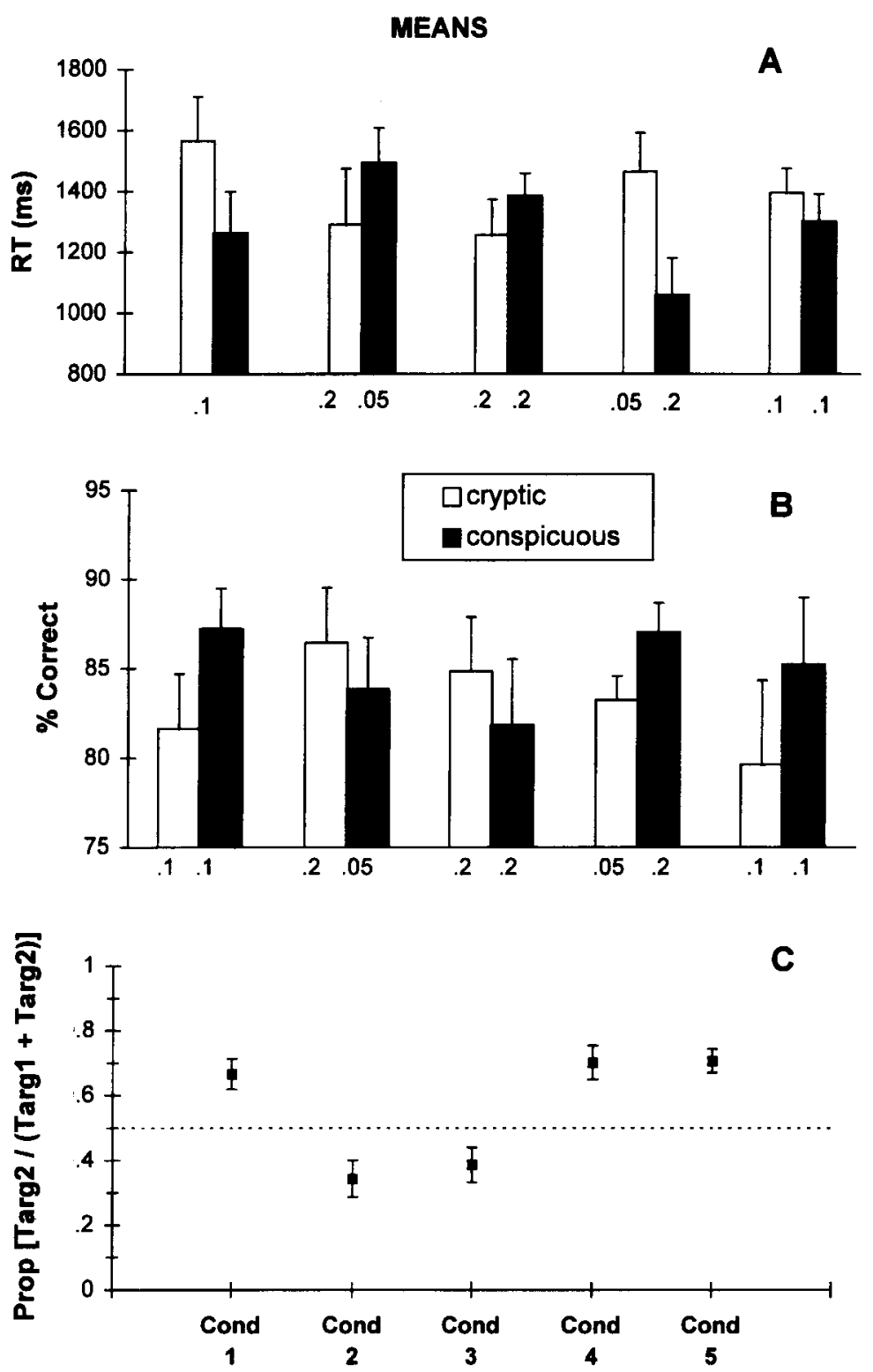

Figure 1. Mean data for the 5 birds in Phase 1 of Experiment 1. The cryptic target (Targ1) was a delta and the conspicuous target (Targ2) was the letter T. Panel $A$ shows mean reaction times (RTs) for each target in each condition. The values below the bars indicate the probability of reinforcement for that target in the condition. Panel $B$ shows mean accuracy in the same way. Panel $C$ shows the mean proportion of correct probe trials in which the conspicuous target (Targ2) was chosen. A proportion of 1.0 means that Targ2 was always chosen on probe trials; a proportion of 0 indicates that Targ 2 was never chosen. The dashed line indicates indifference between the two target items.

dition 2 when the probability of reinforcement for the cryptic target exceeded that for the conspicuous target. As in Phase 1, this reversal carried over to Condition 3 even though the probabilities of reinforcement for both targets were equal and the same for Condition 1. A similar but inverse directional shift can be seen in the mean accuracy data (Figure 2B). The proportion of cryptic targets chosen on probe trials shifted in a corresponding way (Figure $2 \mathrm{C}$ ) but not nearly as much as in Phase 1.
Statistical analyses of Phase 2 data paralleled those for Phase 1. The first analysis tested the effect of condition (i.e., profitability shifts) on the differences between cryptic target (Targ1) and conspicuous target (Targ2) scores. The effects of condition on RT and accuracy were significant $[F(2,8)=5.51 ; F(2,8)=10.57]$. Significant Tukey HSD tests showed that Condition 1 differed from Condition 2 for RT and for accuracy $\left(\alpha=.05, M S_{\mathrm{e}}=\right.$ $\left.169,042.11 ; \alpha=.05, M S_{\mathrm{e}}=4.85\right)$. 

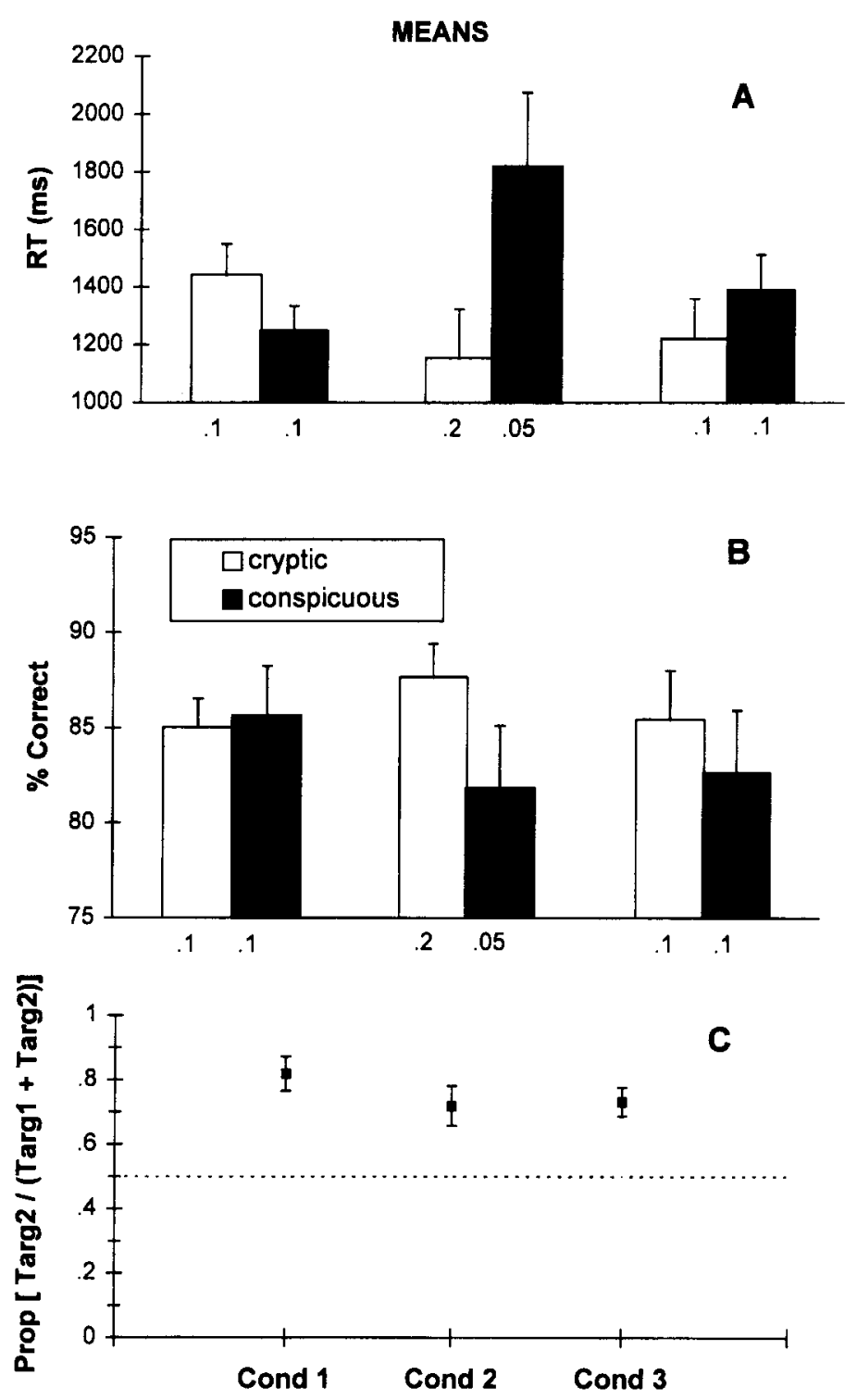

Figure 2. Mean data for all 5 birds in Phase 2 of Experiment 1. The cryptic target (Targ1) was the letter $O$ and the conspicuous target (Targ2) was the letter $\mathbf{E}$. The data are displayed as in Figure 1.

Analysis of the effect of condition on the scores for each individual target type showed a significant effect on RT for target $[F(4,8)=4.78]$. Turkey HSD tests showed that Condition 1 was significantly different from Condition 2 for target RT data $\left(\alpha=.05, M S_{\mathrm{e}}=92,378.9\right)$; other comparisons were not significant.

In summary, changing the relative probability of reinforcement for conspicuous and cryptic targets strongly influenced the speed and accuracy of search for those targets, and it also influenced choice between them in Phase 1. Several important aspects of these effects may be identified. First, increasing the probability of reinforcement for a target increased the speed and accuracy of search for that target, and decreasing the probability of reinforcement for a target decreased the speed of search for that target. Second, under the conditions of this experiment, these effects overrode the effects of target crypticity. Third, these effects of differential reinforcement carried over into conditions of equal reinforcement (Conditions 3 and 5), despite the fact that equal reinforcement was maintained for many sessions and data were reported only after a stability criterion had been met. Possible accounts of these effects of reinforcement probability are considered in the General Discussion section. We consider next some comparable effects of handling cost.

\section{EXPERIMENT 2}

Experiment 1 simulated the manipulation of profitability by variation of prey quality. It demonstrated that dif- 
ferential reinforcement affected search efficiency across targets of differing discriminabilities. Experiment 2 was designed to simulate the manipulation of profitability by variation of handling cost. Discriminability was varied in the same manner as the first experiment. Handling cost was manipulated by changing the number of pecks to the target item required to complete a trial.

\section{Method}

\section{Subjects}

Three of the 5 pigeons used in Experiment 1 served as subjects.

\section{Apparatus and Stimulus Displays}

The apparatus was that used in Experiment 1. The stimulus display was also as in Experiment 1, except that the two target items were an asterisk $\left(^{*}\right)$ and the letter $\mathrm{V}$, and the distractor item was the letter W. In this display, the * was the conspicuous target and the $\mathrm{V}$ was the cryptic target.

\section{Procedure}

The training and testing procedures were in most respects the same as those used in Experiment 1. On any trial, each target had an equal probability (.5) of appearing in the display for all three conditions in this experiment. The total number of trials per session was 625 , the probabilities of reinforcement for each target and probe trial were .1, and the probability of a probe trial occurring was .04 for all three conditions.

The experimental parameter that was manipulated across conditions was the number of pecks that defined a response. In Conditions 1 and 3, this response requirement was three pecks for both target items. In Condition 2, the response requirement was increased to six pecks for the conspicuous target (Targ2) - that is, the letter V. It is important to note that, as in the first experiment, RT was measured to the first peck to an item regardless of the ratio requirement.

The use of a response ratio as high as 6 required a change in the way the ratio was counted. In Experiment 1, the response requirement was an uninterrupted series of pecks to a given screen segment; pecks elsewhere reset the response counter. In Experiment 2, pecks elsewhere did not reset the counter. The reason for this change was that RTs increased dramatically (i.e., greater than $4 \mathrm{sec}$ ), if six pecks in a row to one screen segment were required and RT was measured as the first peck in such a series. Therefore, RT was measured to the first peck in a series of either three or six to one screen segment, with no resetting of RT if a peck was made to a different segment during the series. The correction procedure used in Experiment $I$ was initiated if the bird completed a series of pecks to an incorrect location.

\section{Results and Discussion}

The data are summarized in Figure 3, which shows the mean RT, mean accuracy data, and mean proportion of the conspicuous target taken on probe trials for all 3 birds. The overall session accuracy for 1 of the birds decreased to below $80 \%$ on some of the sessions in this experiment, although it remained above $70 \%$ for all sessions except those involving the cryptic target in Condition 3.

Figure 3 suggests that when the two targets had equal response requirements (Condition 1), the conspicuous target produced a lower RT and higher accuracy than did the cryptic target, and it was chosen more often than the cryptic target on probe trials. When the response requirement was increased for the conspicuous target in Condition 2 , the relation between these measures reversed for the two targets. However, this reversal did not carry over to Condition 3 as it did in Experiment 1.

These suggestions were generally supported by statistical analysis. Because a within-subjects ANOVA is not robust with 3 subjects, planned Tukey HSD tests were used to address specific differences. Tests for the RT and proportion data showed that Condition 2 scores differed significantly from those for Conditions 1 and $3(\alpha=.05$, $M S_{\mathrm{e}}=38,261.74 ; \alpha=.05, M S_{\mathrm{e}}=.00345$ ), and that $\mathrm{RT}$ for Targ2 was significantly longer in Condition 2 than in Conditions 1 and $3\left(\alpha=.05, M S_{\mathrm{e}}=80,271.28\right)$.

In sum, the results of Experiment 2 indicate that a differential response requirement can reverse the effects of crypsis in much the same way as does differential reward (Experiment 1). However, unlike the reward effect in Experiment 1 , the effect of response requirement did not carry over to the subsequent baseline condition in Experiment 2 (Condition 3 ). This suggests that the processes underlying the effects differed somewhat between the two experiments. We consider some possible differences below.

\section{GENERAL DISCUSSION}

In these experiments, we examined the effects of varying the profitability of two prey items of differing crypsis. The main findings were as follows: (1) When the targets were equally profitable, the more discriminable (less cryptic) target yielded faster search and higher accuracy and was taken more often in a choice situation than the less discriminable target. (2) When the relative profitability of the targets was changed by varying the probability of reinforcement, the more profitable target yielded faster search times and higher accuracy and was taken more often in the choice situation. These changes overrode the effects of discriminability on all three measures in Phase 1 and all but choice in Phase 2 of Experiment 1. (3) When the relative profitability was varied by changing the response requirement, the immediate effects were similar to those found by varying probability of reinforcement. (4) The effects produced by differential reinforcement carried over to subsequent equal-probability baseline conditions, while the effects of differential handling cost lasted only while this change was in effect.

Optimal foraging theory predicts that when search cost is low an animal will always take a more profitable item and ignore a less profitable item (Krebs \& McCleery, 1984). This tendency has been confirmed by operant studies in which it was found that animals increase specialization for a more profitable choice item as search cost decreased (Abarca \& Fantino, 1982; Hanson \& Green, 1989; Lea, 1979). Our results are consistent with this finding if one assumes that overall search costs were low, since differences in profitability consistently overwhelmed effects of discriminability. When items have equal profitability, the animal should choose the item with the lower search cost, which is often the target that is easily discriminable or very frequent. In the present study, the birds did in fact choose the more discriminable item when profitability 

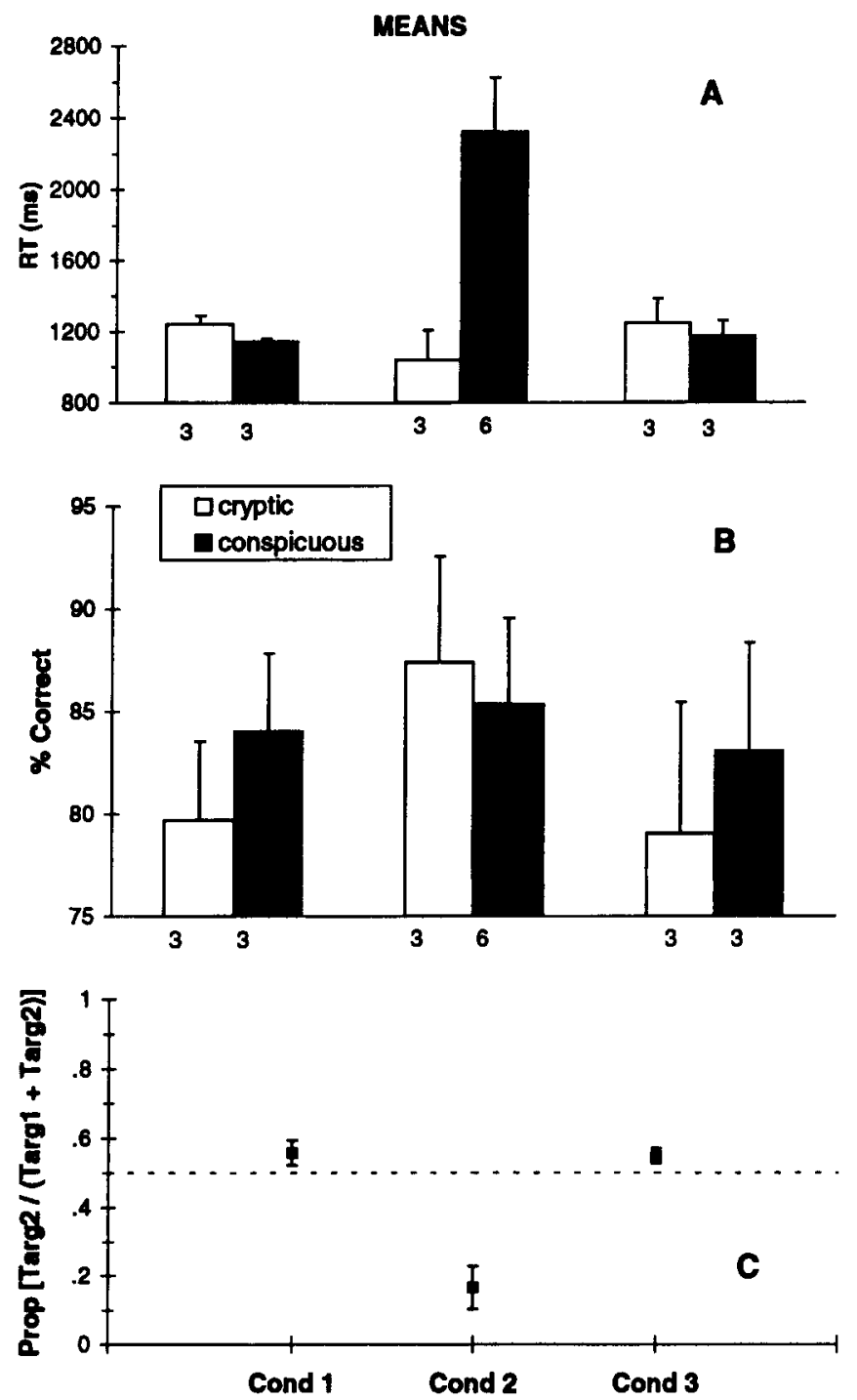

Figure 3. Mean data for the 3 birds in Experiment 2. The cryptic target (Targ1) was the letter $V$, and the conspicuous target (Targ2) was an asterisk (*). The data are displayed as in Figure 1, except that the values below the bars indicate the response requirement as opposed to the probability of reinforcement.

was equal (Condition 1). The finding that greater discriminability results in more efficient search (i.e., faster search and higher accuracy) also confirms previous results (e.g., P. M. Blough, 1992).

In terms of underlying process, the effects induced by changes in profitability associated with the two targets might be traced to changes in one or more of the following: (1) behavioral strategies of search, (2) changes in the relative incentive value of the two targets, or (3) perceptual/ attentional processes. The first of these hypotheses includes suggestions by previous authors that changes in profitability may affect prey handling efficiency or the speed with which search items are examined. Neither of these ideas seems very relevant here. Increased reinforcement or changes in the response ratio of a target might induce a subject to handle that target more efficiently, but "handling" was minimal in the present situation except for the response ratio itself, which the bird could not control. Furthermore, variations in handling cannot easily account for changes in search accuracy or target choice. A more plausible strategic change could be an adjustment of search speed to a level appropriate to finding a particular high-quality item-for example, slowing down when cryptic items become more valuable so that they may be detected more readily (Gendron \& Staddon, 1983, 1984; Guilford \& Dawkins, 1987). This hypothesis also 
accounts for little of the present data. Most obviously, when the more cryptic item was made more valuable, search for that item speeded up, rather than slowing down.

According to the second hypothesis, variations in profitability might affect the incentive values associated with the different targets. Changes in reinforcer amount or probability are well known to affect response speed, accuracy, and choice in other situations. Such effects are traditionally classified as due to changes in incentive. As Logan (1960) comments, incentive might popularly be described as the subject's expectation of reward; just as a rat runs down an alley more rapidly if more food is put in the goalbox, a bird might peck at a highly attractive prey item with relatively high speed. D. S. Blough (1989) found that increasing the probability of reinforcement for one set of targets slowed search for a second set of targets for which reinforcement probability remained unchanged. That result suggested that a target becomes less attractive (i.e., provides a lower incentive) when an alternative target becomes relatively more valuable. Corresponding to this effect is the rise in RT to the less reinforced target at the transition from Condition 1 to Condition 2. However, it is questionable whether incentive changes can account for the entire pattern of data from Experiment 1 . For one thing, Blough failed to find a positive incentive effect; RT to a target did not decrease when that target was associated with more reinforcement (accuracy and choice were not measured). Possibly the incentive effect is reflected primarily by decremental changes in search situations. Also, a sensitivity to the current incentive value of the targets cannot readily explain why differential reinforcement in Condition 2 of each phase influenced search not only in that condition but also throughout the subsequent baseline (Condition 3 ) in which the two targets were equally reinforced for many sessions.

However, incentive shifts might account for the pattern of results of Experiment 2. According to this hypothesis, the relatively high handling cost for one target in Condition 2 reduced the incentive value of that item, and so the bird shifted its choice away from that item and took longer to respond to it. However, when handling costs were again equated, the differential incentive was eliminated, and RT and choice reverted to their previous values.

The third hypothesis suggests that differential reinforcement produces perceptual or attentional changes that improve recognition of the more reinforced target and may degrade recognition of alternative targets. Such a process was proposed by Tinbergen (1960), who suggested that frequent encounters with a food item could generate a "searching image" that would facilitate recognition of that item. Considerable evidence favors this hypothesis; it suggests that a searching image can increase search efficiency for one target and simultaneously decrease it to alternative targets (e.g., P. M. Blough, 1989, 1991; Bond, 1983; Pietrewicz \& Kamil, 1981). Such an effect seems compatible with some of the data reported above. However, the searching image has generally been conceived as a within-session expectancy brought about by repeated encounters or other information about upcoming targets (e.g., P. M. Blough, 1991). Such a shortterm effect does not account for the observed carryover of effects despite many sessions of altered reinforcement conditions. Also, variations in reinforcement probability do not provide the sort of predictive information that the formation of a searching image seems to require.

Though the searching image hypothesis does not predict the present data very effectively, it remains possible that differential reinforcement induces long-term attentional shifts or perceptual learning that act in a somewhat similar way. In this view, the birds learned to focus on the features characteristic of items associated with high reinforcement probability, thus finding those items more efficiently and alternative items less efficiently (Dawkins, 1971 b; Reid \& Shettleworth, 1992). This would account for the observed shifts in response speed, accuracy, and choice that were observed when one target was associated with more frequent reinforcement than was the other. Also, perceptual learning could explain how an originally relatively cryptic target could become detected almost as quickly and accurately as an originally less cryptic target. It is also plausible that a learned tendency to look for specific features might continue until a new arrangement of differential reinforcement caused a refocus on other features; this would account for the observed carryover from conditions of differential reinforcement to subsequent baseline conditions. Finally, given limited processing capacity, a focus on the features of one target implies a less efficient processing of the features of another, and this would help to account for the decrement observed to a target receiving relatively less reinforcement. Overall, then, we find this perceptuallearning or long-term attentional hypothesis a rather compelling account of the observed pattern of data in Experiment 1, though it seems reasonable that incentive changes might have contributed as well. The same account is less persuasive for the results of Experiment 2, since there was no added reinforcement to encourage learning about features and, more importantly, the effect of response requirement failed to carry over beyond the condition in which it was used.

It is important to point out how we suppose the effects of incentive shifts differ from those of perceptual learning. First, we assume that perceptual learning has a longlasting effect while incentive effects are quickly expressed. Second, when there is a choice between two items, an incentive shift would produce a response bias, whereby the most profitable item might be taken even if the less profitable item was found first. In contrast, perceptual learning would cause the more profitable item to be found more efficiently than the less profitable item, but it would not induce a bias against the less profitable item if it was encountered.

On these criteria it is difficult to separate the contributions of perceptual learning and incentive shifts, especially in Experiment 1. However, it appears that an incentive shift by itself can account for the results of Experiment 2 . 
Further research could examine why these two profitability manipulations produced such different results. The incentive hypothesis might be tested through other manipulations, including perhaps the use of qualitatively different reinforcers associated with different targets, together with appropriate motivational shifts (cf. Dickinson \& Balleine, 1994). Also, studies that look at changes in search cost and corresponding changes in response patterns must take into account experience with available prey types, which helps to determine which items are judged most profitable and, consequently, the search strategy used to maximize overall energy gain. This strategy will change when prey types vary within the environment.

A somewhat artificial aspect of this study was that the animals were forced to take items of lower profitability. This was necessary in order to maintain equal encounter rates with both types of items and continued experience with both targets. Thus, the procedure used here ensured that the pigeons would be unable to completely specialize on the most profitable item. Further studies could combine the type of search task used in these studies with the choice component used in operant prey choice tasks (Abarca \& Fantino, 1982; Lea, 1979). In the operant studies, avoidance of a prey was simulated when the bird failed to respond to a keylight for a specified period of time. The use of such a waiting period in a search task could yield a more accurate picture of the cost-benefit relationship. Such a procedure would also help to determine whether an animal simply prefers one item to another or whether the other item is more difficult to detect.

\section{REFERENCES}

ABarCa, N., \& Fantino, E. (1982). Choice and foraging. Journal of the Experimental Analysis of Behavior, 38, 117-123.

BLovgh, D. S. (1989). Contrast as seen in visual search reaction times. Journal of the Experimental Analysis of Behavior, 52, 199-211.

BLough, P. M. (1989). Attentional priming and visual search in pigeons. Journal of Experimental Psychology: Animal Behavior Processes, $15,358-365$.

Blough, P. M. (1991). Selective attention and search images in pigeons. Journal of Experimental Psychology: Animal Behavior Processes, 17, 292-298.

BLough, P. M. (1992). Detectability and choice during visual search: Joint effects of sequential priming and discriminability. Animal Learning \& Behavior, 20, 293-300.

BoND, A. B. (1983). Visual search and selection of natural stimuli in the pigeon: The attention threshold hypothesis. Journal of Experimental Psychology: Animal Behavior Processes, 9, 292-306.

Bond, A. B., \& Riley, D. A. (1991). Searching image in the pigeon: A test of three hypothetical mechanisms. Ethology, 87, 203-224.
Dawkins, M. (1971a). Perceptual changes in chicks: Another look at the "search image" concept. Animal Behaviour, 19, 566-574.

DAwkINS, M. (1971b). Shifts of "attention" in chicks during feeding. Animal Behaviour, 19, 575-582.

Dickinson, A., \& Balleine, B. (1994). Motivational control of goaldirected action. Animal Learning \& Behavior, 22, 1-18.

GENDRON, R. P. (1986). Searching for cryptic prey: Evidence for optimal search rates and formation of search images in quail. Animal Behaviour, 34, 898-912.

Gendron, R. P., \& Staddon, J. E. R. (1983). Searching for cryptic prey: The effect of search rate. American Naturalist, 121, 172-186.

GENDRON, R. P., \& STADDON, J. E. R. (1984). A laboratory simulation of foraging behavior: The effect of search rate on probability of detecting prey. American Naturalist, 124, 407-415.

GUILFORD, T., \& DAWKINS, M. P. (1987). Search images not proven: A reappraisal of recent evidence. Animal Behaviour, 18, 1838-1845.

Hanson, J., \& Green, L. (1989). Foraging decisions: Prey choice by pigeons. Animal Behaviour, 37, 429-443.

Hughes, R. N. (1979). Optimal diets under the energy maximization premise: The effects of recognition time and learning. American Naturalist, 113, 209-221.

KreBs, J. R. (1973). Behavioral aspects of predation. In P. P. G. Bateson \& P. H. Klopfer (Eds.), Perspectives in ethology (pp. 73-111). New York: Plenum.

KreBs, J. R., \& MCCleERY, R. H. (1984). Optimization in behavioral ecology. In J. R. Krebs \& N. B. Davies (Eds.), Behavioral ecology: An evolutionary approach (2nd ed., pp. 91-121). Sunderland, MA: Sinuaer.

LAWRENCE, E. S. (1985a). Evidence for search image in blackbirds (Turdus merula L.): Long-term learning. Animal Behaviour, 33, 1301-1309.

LAWRENCE, E. S. (1985b). Evidence for search image in blackbirds (Turdus merula L.): Short-term learning. Animal Behaviour, 33, 929-937.

LAWRENCE, E. S. (1986). Can great tits (Parus major) acquire search images? Oikos, 47, 3-12.

LEA, S. E. G. (1979). Foraging and reinforcement schedules in the pigeon: Optimal and non-optimal aspects of choice. Animal Behaviour, 27, 875-886.

LoGAN, F. A. (1960). Incentive: How the conditions of reinforcement affect the performance of rats. New Haven, CT: Yale University Press.

Pietrewicz, A. T., \& Kamil, A. C. (1981). Search images and the detection of cryptic prey: An operant approach. In A. C. Kamil \& T. D. Sargent (Eds.), Foraging behavior: Ecological, ethological, and psychological approaches (pp. 311-331). New York: Garland STPM.

REID, P. J., \& SHETTLEWORTH, S. J. (1992). Detection of cryptic prey: Search image or search rate? Journal of Experimental Psychology: Animal Behavior Processes, 18, 273-286.

SCHOENER, T. W. (1987). A brief history of optimal foraging ecology. In A. C. Kamil, J. R. Krebs, \& H. R. Pullium (Eds.), Foraging behavior (pp. 5-67). New York: Plenum.

SHETtLEWORTH, S. J. (1989). Animals foraging in the lab: Problems and promises. Journal of Experimental Psychology: Animal Behavior Processes, 15, 81-87.

TinBERGEN, L. (1960). The natural control of insects in pinewoods: I. Factors influencing the intensity of predation by songbirds. Archives Neerlandaises de Zoologies, 13, 265-343.

(Manuscript received March 7, 1997; revision accepted for publication February 24, 1998.) 